\title{
Laparoscopic resection of a huge gangrenous Meckel's diverticulum in an adult
}

\author{
Marc Weijie Ong $^{1}$, MBBS, Ker Kan $\underline{\operatorname{Tan}}^{1}$, MMed, FRCSE, Richard $\underline{\operatorname{Sim}}^{1}$, MBBS, FRCSE
}

\begin{abstract}
This report highlights the rare occurence of a huge gangrenous Meckel's diverticulum in an adult, which was managed successfully with laparoscopic resection. A 45-year-old woman presented with a one-day history of right iliac fossa pain with fever and vomiting. Computed tomography showed a huge gangrenous Meckel's diverticulum. The patient underwent laparoscopic exploration and extracorporeal stapled resection of the Meckel's diverticulum. This case serves to highlight the safety and feasibility of performing a laparoscopic resection of a huge gangrenous Meckel's diverticulum in an adult.
\end{abstract}

Keywords: adult, gangrene, laparoscopic, Meckel's diverticulum, surgery

\section{INTRODUCTION}

Meckel's diverticulum is regarded as one of the most common congenital anomalies of the gastrointestinal tract. Complications that arise due to a Meckel's diverticulum include bleeding, obstruction, inflammation, and less commonly, perforation. Although complications from a Meckel's diverticulum are rarely seen in the adult population, when observed, emergency surgery is often required. We report an interesting occurence of a huge gangrenous Meckel's diverticulum in an adult patient, which was managed with laparoscopic surgery.

\section{CASE REPORT}

A 45-year-old woman with no significant medical history and no prior surgical history presented with a one-day history of rightsided abdominal pain and vomiting. The pain was a constant, dull ache without any radiation. It was the patient's first episode of abdominal pain that was associated with fever, chills and rigor. She did not have any change in bowel habits and there was no passage of blood per rectum. Physical examination demonstrated tenderness in the right iliac fossa with mild guarding. There was no palpable masses or abdominal distension. She had a raised leucocyte count of $13.6 \times 10^{9} / \mathrm{L}$, but other blood test investigations were unremarkable. The initial impression was that of an acute appendicitis or diverticulitis.

Computed tomography (CT) of the abdomen and pelvis revealed a $6.7 \mathrm{~cm} \times 4.1 \mathrm{~cm} \times 4.1 \mathrm{~cm}$ air-filled mass with dependent debris, suggestive of an infective lesion. The mass appeared to arise from the antimesenteric border of the small bowel, raising suspicion that it may be a Meckel's diverticulum (Fig. 1). A diagnostic laparoscopy was performed in view of the patient's clinical and CT findings. Intraoperatively, we found a large, distended and gangrenous Meckel's diverticulum, measuring $6 \mathrm{~cm} \times 4 \mathrm{~cm}$, which was walled off by the surrounding omentum and small bowel (Fig. 2). The Meckel's diverticulum and ileum

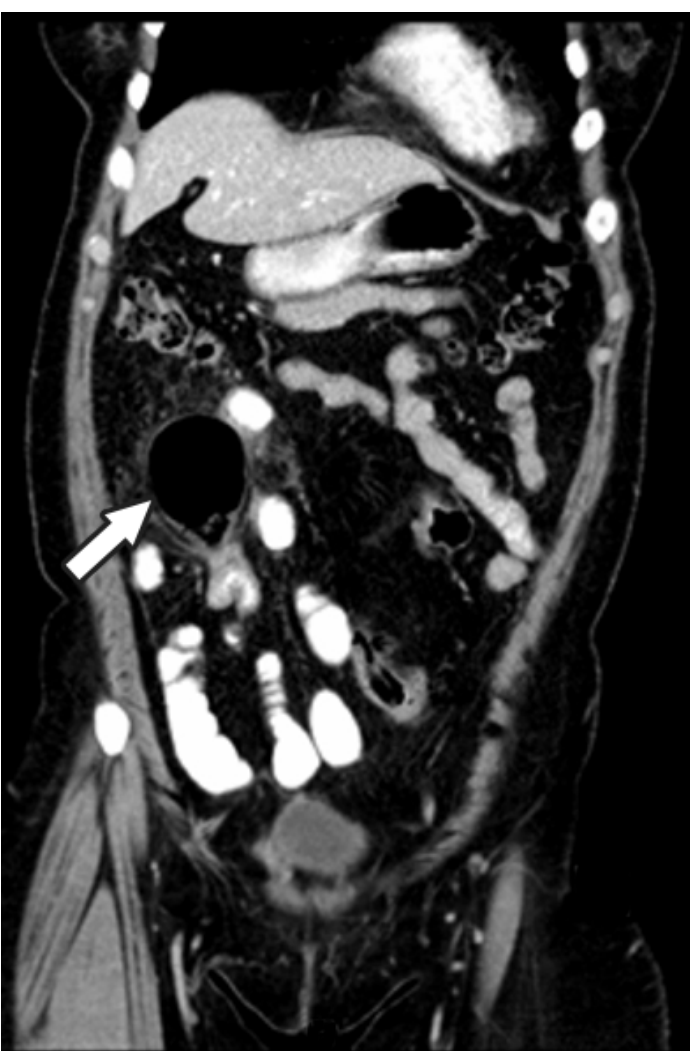

Fig. 1 Coronal CT image of the abdomen and pelvis shows an air-filled mass (arrow), which was suspected to be a Meckel's diverticulum.

were mobile towards the right iliac fossa trocar site. A $2.5 \mathrm{~cm}$ incision was made in the right iliac fossa, and the Meckel's diverticulum with the ileum was brought through an Alexis extra-small wound protector extracorporeally (Fig. 3). A stapled resection of the Meckel's diverticulum was then performed. The patient recovered well and was discharged on the third postoperative day. There were no postoperative complications such as bleeding, infection or formation of intra-abdominal abscess. Pathological results of the specimen confirmed the

${ }^{1}$ Digestive Disease Centre, Department of General Surgery, Tan Tock Seng Hospital, Singapore

Correspondence: Dr Richard Sim, Senior Consultant, Department of General Surgery, Tan Tock Seng Hospital, 11 Jalan Tan Tock Seng, Singapore 308433. richard_sim@ttsh.com.sg 


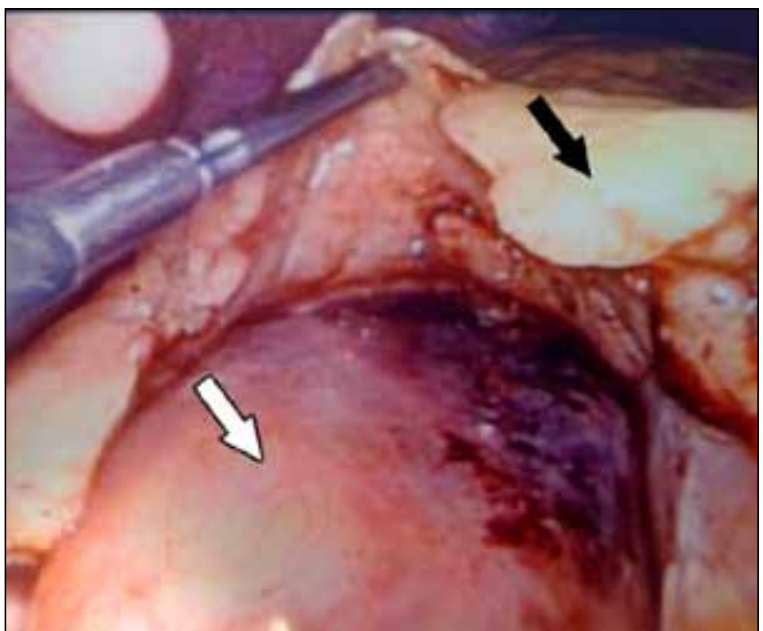

Fig. 2 Intraoperative photograph shows the huge gangrenous Meckel's diverticulum (white arrow), walled off by the surrounding omentum (black arrow).

diagnosis of gangrenous Meckel's diverticulum. The patient was followed up for a period of six months, and there was no recorded incidence of complications such as wound dehiscence, incisional hernia or postoperative adhesive intestinal obstruction.

\section{DISCUSSION}

Preoperative diagnosis of Meckel's diverticulum is a challenge despite the availability of modern imaging. CT and technetium-99m radionuclide imaging are widely used, but the correct diagnosis is often established only at the time of laparotomy or laparoscopy. This is especially the case for Meckel's diverticulum in adults, as symptoms are often nonspecific and complications are likely to occur at the time of presentation. Hence, a diagnosis of symptomatic Meckel's diverticulum demands a high degree of suspicion, as an accurate preoperative clinical diagnosis is often difficult to make.

Although complications due to Meckel's diverticulum are rare, they often present as surgical emergencies. ${ }^{(1,2)}$ These complications often warrant surgical resection of the diseased segment. A gangrenous Meckel's diverticulum is very rare. ${ }^{(3)}$ There are only two previously reported cases of giant gangrenous Meckel's diverticulum caused by axial torsion. ${ }^{(4,5)}$ In both cases, the patients underwent laparotomy, with one patient requiring resection of the involved small bowel with primary anastomosis. This highlights the difficulties encountered when the Meckel's diverticulum becomes gangrenous, as it makes the surgery even more demanding.

As it was clinically difficult to distinguish between diverticulitis and appendicitis in our situation, laparoscopic evaluation was valuable in aiding diagnosis. Excision of the symptomatic

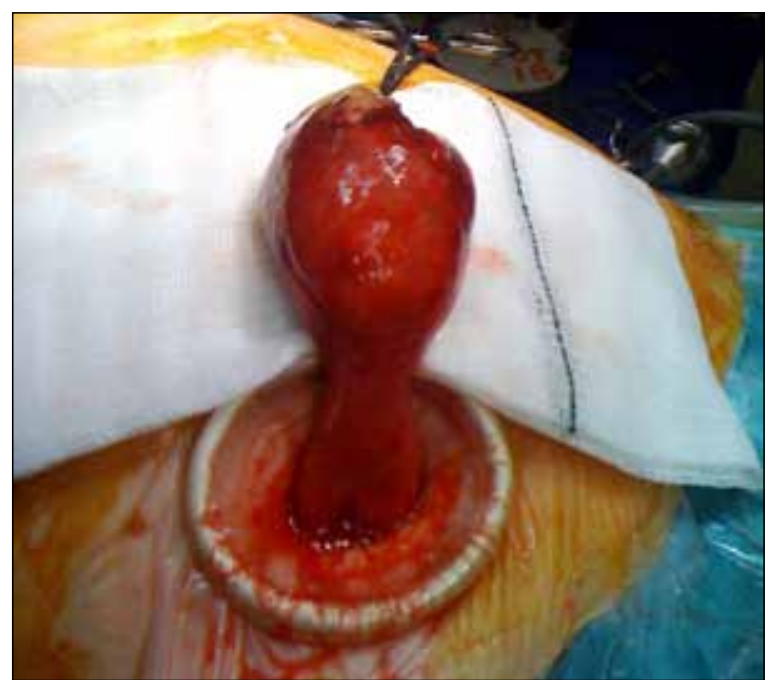

Fig. 3 Intraoperative photograph shows the exteriorised gangrenous Meckel's diverticulum.

Meckel's diverticulum is considered the treatment of choice. With laparoscopy, excision has become minimally invasive, quicker, safer and more efficient. Although intracorporeal resection and anastomosis could have been performed, some of the considerations included spillage from enterotomy and the cost associated with the usage of laparoscopic staplers. ${ }^{(6)}$ In addition, the need for an eventual incision to extract the specimen from our patient made extracorporeal resection a more logical option. The use of a laparoscope allowed a much smaller incision, which upon resection of the diverticulum, enabled the rest of the small bowel to be easily examined.

The routine adoption of laparoscopy in complicated Meckel's diverticulum is not encouraged. Careful patient selection and the laparoscopic experience of the surgeon are important considerations. However, we believe this case serves to highlight the safety and feasibility of performing a laparoscopic resection of a huge gangrenous Meckel's diverticulum in an adult patient.

\section{REFERENCES}

1. Dumper J, Mackenzie S, Mitchell P, et al. Complications of Meckel's diverticula in adults. Can J Surg 2006;49:356-7.

2. Mackey WC, Dineen P. A fifty year experience with Meckel's diverticulum. Surg Gynecol Obstet 1983;156:56-64.

3. Malhotra S, Roth DA, Gouge TH, et al. Gangrene of Meckel's diverticulum secondary to axial torsion: a rare complication. Am J Gastroenterol 1998; 93:1373-5.

4. Nunes QM, Hotouras A, Tiwari S, Sheth A. Gangrene due to axial torsion of a Giant Meckel's Diverticulum containing multiple stones in the lumen: a case report. Cases J 2009; 2:7141.

5. Limas C, Seretis K, Soultanidis C, Anagnostoulis S, et al. Axial torsion and gangrene of a giant Meckel's diverticulum. J Gastrointestin Liver Dis 2006; 15:67-8.

6. Palanivelu C, Rangarajan M, Senthilkumar R, Madankumar MV, Kavalakat AJ. laparoscopic management of symptomatic meckel's diverticula: a simple tangential stapler excision. JSLS 2008; 12:66-70. 